\title{
Proposta alternativa para a estimativa da concentração de carbono em amostras metálicas delgadas de aço-carbono
}

\section{Alternative proposal for the estimation of carbon concentration in thin samples carbon steel}

\author{
Jorge Corrêa de Araújo
} Universidade do Estado do Rio de Janeiro (UERJ), Departamento de Matemática da Faculdade de Formação de Professores (FFP), São Gonçalo, RJ, Brasil jcaraujo 55@yahoo.com.br

Rosa María García Márquez Universidade do Estado do Rio de Janeiro (UERJ), Departamento de Matemática da Faculdade de Formação de Professores (FFP), São Gonçalo, RJ, Brasil rosagmarquez@yahoo.com.br

\begin{tabular}{l}
\hline Informações do Artigo \\
\hline cc) (7) (5) \\
\hline
\end{tabular}

Histórico do Artigo

Submissão: 06 de março de 2018

Aceite: 29 de março de 2018.

Palavras-chave

Difusão de Carbono

Função Erro de Gauss

Polinômio de Aproximação

Interpolação Linear

\begin{abstract}
Resumo
Neste estudo é descrito um processo de cementação de uma amostra metálica homogênea delgada de aço-carbono pela equação de difusão unidimensional. Esse processo é analisado com detalhes à luz da transformada de Fourier em seno para obter a expressão transiente da concentração de carbono em difusão. Uma alternativa aos métodos numéricos envolvidos na resolução da função erro de Gauss, que faz parte da solução formal da equação diferencial, usada para representar esse processo restrito a pequenas profundidades de um sólido, consiste na utilização de um polinômio de quinto grau que possui a vantagem de poder ser utilizado em um domínio contínuo com resultados concordantes com os dos métodos numéricos utilizados. Tal metodologia pode ser estendida a outros solutos em processo de difusão dentro de condições iniciais e de fronteira mais gerais, embora dentro de limites espaciais restritos.
\end{abstract}

\begin{abstract}
In this study, a cementation process of a thin homogeneous carbon steel metal sample is described by one-dimensional diffusion equation and analyzed in details at the light of the Fourier transform in sine to obtain the transient expression of the carbon concentration in diffusion. An alternative to the numerical methods involved in solving the Gauss error function which is part of the formal solution of the differential equation that is used to represent this process, although restricted to thin samples consists in the use of a fifth degree polynomial that has the advantage of being able to be used in a continuous domain with results concordant with those of the numerical methods used. Such methodology can be extended to other diffusive solutes within more general initial and boundary conditions, though within restricted spatial limits.
\end{abstract}

\section{Introdução}

A equação de difusão em regime transiente em um meio unidimensional, com coeficiente de difusão constante é bem conhecida, sendo dada por $\frac{\partial C}{\partial t}=D \frac{\partial C^{2}}{\partial x^{2}}$. Esta equação também é conhecida como a Segunda Lei de Fick (GARZÓN; TSCHIPTSCHIN, 2006; TIJONOV; SAMARSKY, 1980). Nessa equação, $C(x, t)$ representa a concentração de um soluto em um ponto específico de um meio difusivo em um determinado instante de tempo, como por exemplo, um ponto situado 
no interior de um sólido a uma distância, $x$ da superfície. A constante é denominada coeficiente de difusão, e dá uma medida da taxa na qual os átomos do soluto se difundem no interior do sólido.

Durante esse processo, a concentração do elemento em difusão pode aumentar ou se esgotar ao longo do tempo. Obter, portanto, estimativas de concentrações desejadas de determinado soluto em processos de engenharia é de fundamental importância para garantir que certas propriedades em determinado tipo de material sejam alcançadas. Uma das aplicações mais importantes de difusão em engenharia é o processo de cementação também conhecido como carbonetação, que consiste no endurecimento da superfície de um aço para níveis mais elevados do existente em seu interior pela difusão de átomos de carbono na mesma superfície, quando exposta em um ambiente rico em carbono em altas temperaturas (CALLISTER, 2002).

Para a representação desse processo, por meio da equação de difusão transiente, é preciso estabelecer condições iniciais e de fronteira adequadas, de modo que tenham sentido físico plausível e que a solução única possa ser obtida. Normalmente, soluções para esse tipo de equação são obtidas por meio de variáveis separáveis (BOYCE; DIPRIMA, 1999), ou soluções numéricas (ARAÚJO; MÁRQUEZ, 2013). Mas também podem ser obtidas usando a transformada de Fourier em seno como foi proposto por Debnath e Bhatta (2015), considerando a concentração inicial nula ou usando a transformada de Laplace como foi adotado por Crank (2011).

Neste artigo será considerada uma placa metálica delgada homogênea de ferro-carbono, ou $\mathrm{Fe}_{\gamma}-C$, submetida a um processo de cementação, onde a condição inicial é dada por $C(x, 0)=C_{0}$, com $0 \leq x<\infty$, e a condição superficial dada como $C(0, t)=C_{1}$, com $t>0$ sendo $C_{1}$ uma constante positiva qualquer. Outras condições iniciais com soluções abrangentes, mas não detalhadas podem ser vistas no livro escrito por Carslaw e Jaeger (2011).

A solução para o problema analisado neste estudo foi obtida por meio da transformada de Fourier em seno considerando a concentração inicial não nula e a solução obtida detalhadamente depende da função erro de Gauss $\operatorname{erf}(z)$ (DEBNATH; BHATTA, 2015). A vantagem do uso da transformada de Fourier em seno é que ela reduz a equação diferencial parcial que é utilizada para representar a difusão unidimensional transiente em uma equação diferencial ordinária linear de primeira ordem, embora no processo da transformação, integrais de difícil tratamento matemático possam ocorrer. Esse método foi utilizado por Negero (2014) para obter a solução formal da equação de Laplace em um quarto de plano entre outros exemplos analisados. Castro (2014) usou as transformadas de Fourier em seno e cosseno para estudar um potencial duplo com base na equação de Schrödinger. Variados exemplos da aplicação da transformada de Fourier em seno e cosseno são também apresentados por Trim (1990) envolvendo equações diferenciais parciais em problemas de valores de fronteira.

Uma alternativa ao uso direto da integral $\operatorname{erf}(z)$, que é calculada em geral por meio de uma interpolação linear dos dados tabelados, ou fazendo o uso de métodos de integração numérica, foi 
desenvolvida nesse estudo para ser utilizada em um domínio espacial contínuo com o tempo fixado. Os resultados mostram que os níveis de concentração de carbono na placa durante o processo de cementação usando o método alternativo aqui proposto para o cálculo da integral erro de Gauss ficaram em boa concordância com o uso da integral erro de Gauss calculada por meio de tabelas ou métodos numéricos em placas de espessura menor ou igual a dois milímetros.

\section{Materiais e Métodos}

Considere uma placa metálica homogênea formada por uma liga de Carbono em Ferrogama, indicada por, $\mathrm{Fe}_{\gamma}-\mathrm{C}$ que deve ser "endurecida" através de um processo de cementação (CALLISTER, 2002). A peça de aço é exposta, em uma atmosfera rica em hidrocarboneto gasoso (como o gás metano, $\mathrm{CH}_{4}$ ), sob a temperatura $\mathrm{T}=1000^{\circ} \mathrm{C}$. A placa homogênea, com as propriedades da Tabela 1, contém uma concentração uniforme de carbono de $0,20 \% p C$ (percentual em peso de carbono). A concentração de carbono é mantida constante na superfície da placa em $1,30 \% p C$. Nessas condições, o objetivo é obter o tempo necessário durante o processo de cementação para que na profundidade, $x_{L}=0,002 m$ se tenha, por exemplo, $0,45 \% p C$. A Figura 1 mostra de forma simplificada a placa metálica sujeita às condições dadas no processo de cementação descritas como anteriormente.

Figura 1 - Difusão transiente de carbono através de uma placa de aço delgada de ferro carbono.

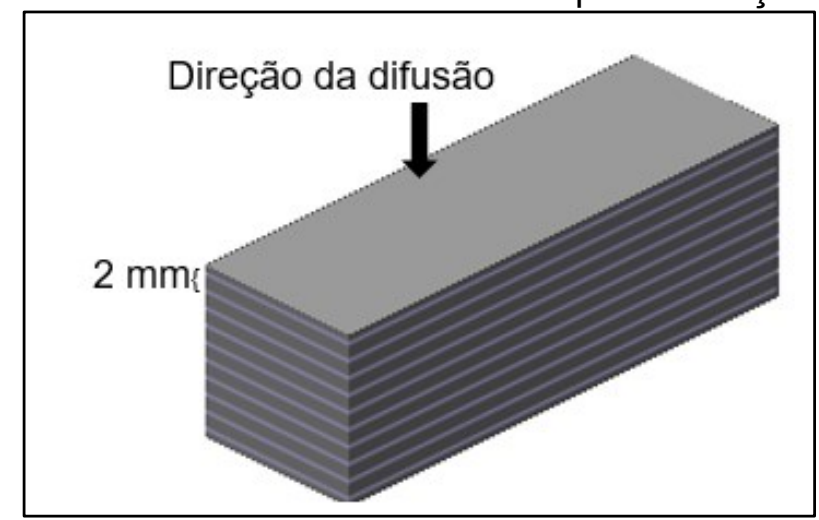

Fonte: Elaboração dos autores.

Na Tabela 1 são apresentados os parâmetros do processo de cementação, notação e valores assumidos e as condições iniciais do processo de difusão. 
Tabela 1 - Parâmetros considerados no processo de cementação.

\begin{tabular}{|c|c|c|}
\hline Parâmetros do processo & Notação & Valores \\
\hline Espessura da placa & $x_{L}$ & $0,002 \mathrm{~m}$ \\
\hline Difusividade & $D_{o}$ & $2,3 \times 10^{-5} \mathrm{~m}^{2} / \mathrm{s} *$ \\
\hline Concentração inicial de C & $C(x, 0)=C_{o}$ & $0,20 \% \mathrm{C}$ \\
\hline Concentração superficial de C & $C(0, t)=C_{1}$ & $1,30 \% p C$ \\
\hline Temperatura de difusão & $T$ & $100{ }^{0} \mathrm{C}_{*}$ \\
\hline Energia de ativação & $Q d$ & $148 \mathrm{~kJ} / \mathrm{mol}_{*}$ \\
\hline Constante dos gases & $R$ & $8,31 \mathrm{~J} / \mathrm{mol}-\mathrm{K}_{*}$ \\
\hline Densidade do Ferro gama & $\rho_{F e \gamma}$ & $7,86 \mathrm{~g} / \mathrm{cm} 3^{* *}$ \\
\hline Densidade do carbono & $\rho_{C}$ & $2,267 \mathrm{~g} / \mathrm{cm}^{* * *}$ \\
\hline
\end{tabular}

*Valor obtido de referência para essa difusão (CALLISTER, 2002, p. 70, Tabela 5.2).

**Valor obtido de referência para essa difusão (CALLISTER, 2002, p. 537, Tabela B.1).

${ }^{* *}$ https://iupac.org/. Union of Pure and Applied Chemistry (IUPAC).

\section{A Transformada de Fourier em Seno}

Teorema 1. Seja $f(x)$ uma função seccionalmente contínua sobre todo o intervalo finito no eixo $x$ e que nos pontos de descontinuidade, $x_{0}$, é definida como $\frac{1}{2}\left[f\left(x_{0}^{+}\right)+f\left(x_{0}^{-}\right)\right]$e que seja absolutamente integrável. Então, em todo ponto $x$ onde $f(x)$ é derivável, a função $f(x)$ é representada pela fórmula integral de Fourier (CHURCHILL, 1963)

$$
f(x)=\frac{1}{\pi} \int_{0}^{\infty} \int_{-\infty}^{\infty} f(\xi) \cos [\alpha(\xi-x)] d \xi d \alpha, \quad-\infty<x<\infty
$$

onde $x, \alpha \in I R$.

Suponhamos também que $f(x)$ é uma função ímpar. Daí, $f(\xi) \operatorname{sen}(\alpha \xi)$ é uma função par e é possível mostrar (CHURCHILL, 1963, p. 118) que

$$
f(x)=\frac{2}{\pi} \int_{0}^{\infty} \operatorname{sen}(\alpha x)\left(\int_{0}^{\infty} f(\xi) \operatorname{sen}(\alpha \xi) d \xi\right) d \alpha
$$

onde a equação (2) é denominada fórmula integral de Fourier em seno. Considerando $f(x)$ com as condições anteriormente assumidas no Teorema 1 a sua transformada de Fourier em seno, $\mathbf{F}_{s}(\alpha)$ (ou $\left.\mathbf{F}_{s}\{f\}(\alpha)\right)$ de $f(x)$ é dada, por

$$
\mathrm{F}_{s}(\alpha)=\int_{0}^{\infty} f(\xi) \operatorname{sen}(\alpha \xi) d \xi
$$

enquanto, a fórmula de inversão, ou transformada inversa de Fourier em seno, $\mathbf{F}_{s}^{-1}\left\{\mathbf{F}_{s}(\alpha)\right\}$ é dada por

$$
f(x)=\frac{2}{\pi} \int_{0}^{\infty} \mathrm{F}_{s}(\alpha) \operatorname{sen}(\alpha x) d \alpha
$$




\subsection{Propriedades}

1) Sejam $f$ e $g$ funções integráveis. As transformadas de Fourier em seno e cosseno são lineares, isto é,

$$
\mathbf{F}_{s}\{a f(x)+b g(x)\}=a \mathbf{F}_{s}\{f(x)\}+b \mathbf{F}_{s}\{g(x)\}
$$

onde $a$ e $b$ são duas constantes reais.

2) Suponhamos que $f, f^{\prime}$ e $f^{\prime \prime}$ são funções contínuas para todo $x \geq 0$ e que $f, f^{\prime} \rightarrow 0$ quando $x$ tende ao infinito. Se, além disso, fé absolutamente integrável, isto é, $\int_{0}^{\infty}|f(x)| d x<\infty$, então

$$
\mathbf{F}_{s}\left\{f^{\prime \prime}\right\}(\alpha)=-\alpha^{2} \mathbf{F}_{s}(\alpha)+\alpha f(0)
$$

A propriedade (2) mostra que a transformação do seno troca a forma diferencial $f^{\prime \prime}(x)$ por uma equação algébrica envolvendo $\mathbf{F}_{s}(\alpha)$, e o valor inicial $f(0)$. Segundo lório (1991), Debnath e Bhatta (2015) e Churchill (1963), as propriedades (1) e (2) conduzem à redução de certos tipos de problema de valores de fronteira em simples problemas de transformadas de funções desconhecidas como é visto nesse trabalho. Para lidarmos com as integrais impróprias que aparecem na transformada de Fourier em seno, daremos a seguir alguns resultados de análise complexa.

Teorema 2. Teorema dos Resíduos de Cauchy. Seja $C$ um caminho fechado simples orientado positivamente. Se $f$ é uma função analítica em cada ponto de $C$ e em todos os pontos da região limitada por $C$, exceto por um número finito de singularidades $z=z_{k}(k=1,2, \ldots, n)$, então (BROWN; CHURCHILL, 2015, p. 233).

$$
\int_{C} f(z) d z=2 \pi i \sum_{k=1}^{n} \operatorname{Res}\left(f(z), z_{k}\right)
$$

Caso $f$ tenha somente uma singularidade simples na origem, então pelo teorema de Cauchy para os resíduos (BROWN; CHURCHILL, 2015, p. 80) tem-se

$$
\int_{-\infty}^{\infty} \frac{e^{i x \xi}}{\xi} d \xi=2 \pi i \sum_{\operatorname{Im} w_{k}>0} \operatorname{Res}\left(\frac{e^{i x w}}{w}, w_{k}\right)+\pi i \sum_{\operatorname{Im} w_{j}=0} \operatorname{Res}\left(\frac{e^{i x w}}{w}, w_{j}\right)
$$

Teorema 3. Seja $f(z)$ uma função analítica em cada ponto do semiplano superior $y \geq 0$, exterior ao semicírculo de equação $|z|=R_{0}$ e $C_{R}$ a curva $C_{R}=\left\{z / z=\operatorname{Re}^{i \theta} \quad 0 \leq \theta \leq \pi ; R>R_{0}\right\}$. Se existe 
alguma constante $M_{R}$ tal que $|f(z)| \leq M_{R}$ e $\lim _{R \rightarrow \infty} M_{R}=0$ para todo ponto $z \in C_{R}$, então, dada qualquer constante positiva $a$, temos que $\lim _{R \rightarrow \infty} \int_{C_{R}} f(z) e^{i a z} d z=0$.

\section{Resultados e Discussões}

A equação que será usada para representar a concentração, $C=C(x, t)$, no problema proposto no processo de cementação é dada por Callister (2002, p. 67) como

$$
\frac{\partial C}{\partial t}=D \frac{\partial C^{2}}{\partial x^{2}} ; x, t>0
$$

e sujeita às condições inicial e de fronteira dadas respectivamente por

$$
\begin{gathered}
C(x, 0)=C_{0}, 0 \leq x<\infty \\
C(0, t)=C_{1}, t>0
\end{gathered}
$$

com $C=C_{0}$, quando $x$ tende ao infinito. A condição (11) de Dirichlet (IÓRIO, 1991) afirma que a concentração de soluto na superfície do metal deve ser mantida constante, igual a $C_{0}$, enquanto durar o processo. Aplicando a transformada $\mathbf{F}_{s}(\xi)$ em relação à variável x na equação (9) e usando a equação (6) tem-se

$$
\frac{d}{d t} C_{s}(\xi, t)+D \xi^{2} C_{s}(\xi, t)=D \xi C_{1}
$$

onde $C_{s}(\xi, t)=\mathbf{F}_{s}\{C(x, t)\}$. Aplicando novamente $\mathbf{F}_{s}(\xi)$ na condição (10) e usando o fato que a transformada de Fourier em seno de uma função constante $k$ é igual a

$$
\mathrm{F}_{s}\{k\}=\frac{k}{\xi}
$$

tem-se que

$$
\mathrm{F}_{s}\left\{C_{0}\right\}=\frac{C_{0}}{\xi}
$$

Assim, das equações (12) e (14) para cada $\xi \in I R$ fixo, temos um problema de valor inicial para uma equação diferencial linear de primeira ordem em $t$, a qual pode ser resolvida usando, por exemplo, o método do fator integrante (BOYCE; DIPRIMA, 1999) e desse modo obter

$$
C_{s}(\xi, t)=\left(C_{0}-C_{1}\right) \frac{1}{\xi} e^{-D t \xi^{2}}+\frac{C_{1}}{\xi} .
$$

Da equação (15) usando a transformada inversa de Fourier em seno (equação 4) tem-se

$$
C(\xi, t)=\frac{2}{\pi}\left(C_{0}-C_{1}\right) \int_{0}^{\infty} \frac{1}{\xi} e^{-D t^{2}} \operatorname{sen}(\xi t) d \xi+\frac{2}{\pi} C_{1} \int_{0}^{\infty} \frac{\operatorname{sen}(\xi t)}{\xi} d \xi .
$$


Como um dos objetivos deste estudo é conseguir obter com detalhes a solução formal para a equação (16), vamos então destacar as integrais impróprias que compõem a equação da concentração transiente, $C(\xi, t)$ para calculá-las separadamente. Tais integrais aparecem em textos especializados, como o de Debnath e Bhatta (2015), onde são apresentadas de forma quase direta, isto é, sem qualquer detalhamento de suas soluções.
a) $\int_{0}^{\infty} \frac{\operatorname{sen}(\xi t)}{\xi} d \xi$
b) $\int_{0}^{\infty} \frac{1}{\xi} e^{-D t \xi^{2}} \operatorname{sen}(\xi t) d \xi$

Para o cálculo da integral (a) utilizamos a seguinte metodologia. Considere a função complexa

$$
f(z)=\frac{z}{z^{2}+b^{2}}
$$

Multiplicando ambos os lados da equação (17) pelo fator $e^{i \alpha z}$ têm-se

$$
f(z) e^{i \alpha z}=\frac{z e^{i \alpha z}}{z^{2}+b^{2}}=\frac{\phi(z)}{z-i b} .
$$

Da equação (18) resulta que

$$
\phi(z)=\frac{z e^{i \alpha z}}{z+i b}
$$

A função $\phi(z)$ é analítica e não nula no polo $z_{o}=i b$ que é uma singularidade de $f(z) e^{i \alpha z}$. Essa singularidade encontra-se no interior da região limitada cuja fronteira consiste no segmento, $-R \leq x \leq R$ (com $R>\left|z_{o}\right|=\sqrt{b}$ ) do eixo real e a metade superior do círculo $|z|=R$ de $z=-R$ até $z=R\left(C_{R}\right)$, tornando o caminho orientado positivamente. Usando o Teorema 2 (Teorema dos resíduos de Cauchy), segue que

$$
\lim _{R \rightarrow \infty} \int_{-R}^{R} \frac{x e^{i \alpha x}}{x^{2}+b^{2}} d x=2 \pi i \phi\left(z_{o}\right)-\int_{C_{R}} f(z) e^{i \alpha z} d z
$$

onde

$$
\operatorname{Res}\left(f(z) e^{i \alpha z}, z=z_{o}\right)=\phi\left(z_{o}\right)=\frac{e^{-\alpha b}}{2}
$$

Se $z \in C_{R}, \quad\left|z^{2}+b^{2}\right| \geq|z|^{2}-b^{2} \mid=R^{2}-b^{2}$, fazendo a inversão da desigualdade tem-se que

$$
\frac{1}{\left|z^{2}+b^{2}\right|} \leq \frac{1}{R^{2}-b^{2}}
$$


observe que

$$
\left|e^{i \alpha z}\right|=\left|e^{i \alpha(x+i y)}\right|=e^{-\alpha y}\left|e^{i \alpha x}\right| \leq e^{-\alpha R},
$$

logo, das desigualdades (22) e (23) segue que

$$
\left|f(z) e^{i \alpha z}\right|=\left|\frac{z e^{i \alpha z}}{z^{2}+b^{2}}\right| \leq \frac{\mathrm{Re}^{-\alpha R}}{R^{2}-b^{2}}=M_{R}
$$

Então considerando as partes imaginárias da equação (20) resulta

$$
\int_{-R}^{R} \frac{x \operatorname{sen}(\alpha x)}{x^{2}+b^{2}}=\pi e^{-\alpha b}-\operatorname{Im} \int_{C_{R}} f(z) e^{i \alpha z} d z
$$

Da desigualdade (23) e do Teorema 2, tem-se que $\lim _{R \rightarrow \infty} \int_{C_{R}} f(z) e^{i \alpha z} d z=0$. Daí, e da equação (25) obtemos

$$
\int_{-\infty}^{\infty} \frac{x \operatorname{sen}(\alpha x)}{x^{2}+b^{2}} d x=\pi e^{-\alpha b}
$$

ou, usando a paridade do integrando,

$$
\int_{0}^{\infty} \frac{x \operatorname{sen}(\alpha x)}{x^{2}+b^{2}} d x=\frac{\pi}{2} e^{-\alpha b}
$$

Ao fazer $b=0$ na equação (27), e como $x>0$, tem-se que

$$
\int_{0}^{\infty} \frac{\operatorname{sen}(\alpha x)}{x} d x=\frac{\pi}{2}
$$

Fazendo uma troca nas variáveis, pode-se obter a solução da integral procurada da integral (I), isto é,

$$
\int_{0}^{\infty} \frac{\operatorname{sen}(\alpha x)}{\alpha} d \alpha=\frac{\pi}{2}
$$

Para o cálculo da integral (b) usaremos uma adaptação do resultado proposto por Debnath e Bhatta (2015) para obter

$$
\mathbf{F}_{s}\left\{\operatorname{erf}_{c}\left(\frac{x}{2 a}\right)\right\}=\frac{1}{\xi}-\frac{1}{\xi} e^{-a^{2} \xi^{2}}
$$

onde $a$ é uma constante não nula e

$$
\operatorname{erf}_{c}\left(\frac{x}{2 a}\right)=1-\operatorname{erf}\left(\frac{x}{2 a}\right)
$$

Sendo

$$
\operatorname{erf}(y)=\frac{2}{\sqrt{\pi}} \int_{0}^{y} e^{-\xi^{2}} d \xi
$$


a função erro de Gauss (CHURCHILL, 1963) e $\operatorname{erf}_{c}\left(\frac{x}{2 a}\right)$ a função erro complementar. Aplicando a transformada de Fourier em seno na equação (30) tem-se

$$
\mathbf{F}_{s}\left\{\operatorname{erf}\left(\frac{x}{2 a}\right)\right\}=\mathbf{F}_{s}\{1\}-\mathbf{F}_{s}\left\{\operatorname{erf}_{c}\left(\frac{x}{2 a}\right)\right\}
$$

ou, das equações (30), (31) e (33) tem-se

$$
\mathbf{F}_{s}\left\{\operatorname{erf}\left(\frac{x}{2 a}\right)\right\}=\frac{1}{\xi} e^{-a^{2} \xi^{2}}
$$

Finalmente, aplicando a transformada inversa de Fourier em seno, $\mathbf{F}_{s}^{-1}$ na equação (34) obtemos, fazendo, $a=\sqrt{D t}$, o resultado da integral (b)

$$
\int_{0}^{\infty} \frac{1}{\xi} e^{-D t \xi^{2}} \operatorname{sen}(\xi t) d \xi=\frac{\pi}{2} \operatorname{erf}\left(\frac{x}{2 \sqrt{D t}}\right)
$$

Das equações (16), (28) e (35) obtemos a expressão para $C(x, t)$, dada por

$$
C(x, t)=\left(C_{0}-C_{1}\right) \operatorname{erf}\left(\frac{x}{2 \sqrt{D t}}\right)+C_{1}
$$

ou, ainda como

$$
\frac{C(x, t)-C_{0}}{C_{1}-C_{0}}=1-\operatorname{erf}\left(\frac{x}{2 \sqrt{D t}}\right)
$$

onde D é o coeficiente de difusão (CALLISTER, 2002). Caso se deseje atingir certa concentração do soluto, $C_{2}$ em uma liga, em um determinado instante de tempo $t_{1}$ e na profundidade $x$, $C_{2}=C\left(x, t_{1}\right)$ teremos da equação (37) que

$$
\frac{C_{2}-C_{0}}{C_{1}-C_{0}}-1=k_{1}
$$

e

$$
\frac{x}{2 \sqrt{D t_{1}}}=z_{0}
$$

sendo $\operatorname{erf}\left(z_{0}\right)=-k_{1}$, sendo $z_{0}$ uma constante real positiva. O valor para $\operatorname{erf}\left(z_{0}\right)$ pode ser obtido por interpolação linear dos dados tabelados da função erro, e desse modo, é possível obter uma estimativa para o tempo, $t_{1}$ necessário para que a concentração $C_{2}$ do soluto seja alcançada.

O cálculo da concentração, $C(x, t)$ usando a equação (36) requer, como foi visto, dados tabelados da função $\operatorname{erf}(z)$ ou então, o seu cálculo por algum método numérico de integração, o que constitui uma importante restrição quando se deseja analisar um conjunto apreciável de concentrações. Escrevendo a equação (36) na forma integral tem-se 


$$
C(x, t)=C_{1}+\frac{2}{\sqrt{\pi}}\left(C_{0}-C_{1}\right) \int_{0}^{\frac{x}{2 \sqrt{D t}}} e^{-y^{2}} d y .
$$

A integral que aparece no lado direito da equação (40) pode ser resolvida por algum método numérico, como por exemplo, a regra $1 / 3$ de Simpson repetida (RUGGIERO; LOPES, 2006). Todavia, a natureza de um método numérico utiliza um domínio discreto na variável espacial, embora, na maioria dos casos é o suficiente para aplicações em engenharia. Visando contornar em parte tais limitações, isto é, fazendo uso de tabelas ou via integração numérica da função erro, propomos, neste estudo, a aproximação da função, $f(y)=e^{-y^{2}}$ por uma expansão em série de Taylor até a quarta ordem (LIMA, 2016) em torno da origem. Desse modo, podemos escrever

$$
\operatorname{erf}(z) \cong p_{5}(z)
$$

onde

$$
p_{5}(z)=\frac{2}{\sqrt{\pi}}\left(z-\frac{1}{3} z^{3}+\frac{1}{3} z^{5}\right)
$$

é um polinômio de quinto grau, com $z=\frac{x_{L}}{2 \sqrt{D t_{1}}}$.

Das equações (40) e (42) tem-se que a concentração estimada por esse método, $C_{p_{5}}\left(x, t_{1}\right)$ é dada por

$$
C_{p_{5}}\left(x, t_{1}\right)=C_{1}+\frac{2}{\sqrt{\pi}}\left(C_{0}-C_{1}\right)\left[\frac{x}{2 \sqrt{D t_{1}}}-\frac{x^{3}}{24 D t_{1} \sqrt{D t_{1}}}+\frac{x^{5}}{320\left(D t_{1}\right)^{2} \sqrt{D t_{1}}}\right]
$$

com $0 \leq x \leq x_{L}$, no tempo fixo $t_{1}$, onde $t_{1}$ pode ser determinado por meio da equação (39) para cada $x_{L} \leq 0,002(m)$ também fixado. A Tabela 2 mostra a tabulação de dois valores da função erro que serão utilizados para obter uma estimativa para o tempo $t_{1}$ para que a concentração de carbono na posição $x_{L}=0,002(m)$ atinja $0,45 \%$ pC, usando a interpolação linear.

Tabela 2 - Tabulação de valores da $\operatorname{erf}(z)$.

\begin{tabular}{|c|c|}
\hline$z$ & $\operatorname{erf}(z)$ \\
\hline 0,85 & 0,77066 \\
\hline$z_{0}$ & 0,77270 \\
\hline 0,90 & 0,79691 \\
\hline
\end{tabular}

Fonte: Adaptado de Callister (2002, p. 68).

Os dados apresentados na Tabela 2 com respeito a primeira e terceira linha foram extraídos de Callister (2002, p. 68). A Tabela 2 foi utilizada tendo em vista a obtenção da constante $\mathrm{k}_{1}$ por meio das equações (38) e (39) e considerando os dados da Tabela 1 para obter $\operatorname{erf}\left(z_{0}\right)=-k_{1}=0,7727$, onde $C_{2}=0,45 \% p C$ é a concentração que deve ser obtida na profundidade $x=x_{L}=0,002(m)$. 
Fazendo uma interpolação linear com os dados da Tabela 2 obtemos $z_{0}=0,85372$, que substituído na equação (39) e considerando os parâmetros (da Tabela 1) $x=x_{L}=0,002(m)$ e o coeficiente $D$ aproximado (CALLISTER, 2002, p. 70) para esse tipo de difusão dado por $D=1,93 \times 10^{-11}\left(\mathrm{~m}^{2} / \mathrm{s}\right)$ resulta no tempo $t_{1}=19,7(h)$.

Por simplicidade é assumido $t_{1}=20(h)$ como o tempo necessário para que o teor de $0,45 \%$ $p C$ seja alcançado no limite da placa. A Tabela 3 mostra estimativas para $C\left(x, t_{1}\right)$ obtidas por meio da interpolação linear, à regra de Simpson (usando quarenta e quatro pontos) e o polinômio de quinto grau, $p_{5}(z)$ aqui adotado como uma metodologia alternativa a esses métodos.

Tabela 3 - Estimativas para $C\left(x, t_{1}\right)$ em \% $p C$ com os métodos analisados.

\begin{tabular}{|c|c|c|c|}
\hline Posição & Regra de Simpson & Interpolação & Polinômio \\
\hline$x(m)$ & $C_{S}$ & $C_{I}$ & $C_{p_{5}}$ \\
\hline 0,000125 & 0,01234 & 0,01230 & 0,01230 \\
\hline 0,000250 & 0,0116 & 0,01160 & 0,01160 \\
\hline 0,0005 & 0,0104 & 0,0104 & 0,0104 \\
\hline 0,001 & 0,008 & 0,008 & 0,008 \\
\hline 0,002 & 0,0045 & 0,0048 & 0,0044 \\
\hline
\end{tabular}

Fonte: Dados da pesquisa.

A Tabela 3 mostra que não existem diferenças significativas entre a metodologia alternativa aqui proposta usando o polinômio, $p_{5}(z)$ em relação à regra de Simpson e à interpolação linear. Diante da concordância dos resultados obtidos com os diferentes métodos listados na Tabela 3, e devido ao maior esforço de lidar-se com a interpolação, usaremos a partir de agora as concentrações estimadas pela regra de Simpson e o polinômio de quinto grau $p_{5}(z)$. A Figura 2 mostra o gráfico contínuo, $C_{p_{5}}\left(x, t_{1}=20 h\right)$ em comparação com as concentrações discretas estimadas usando a regra de Simpson, $C_{S}\left(x_{i}, t_{1}=20\right)$, onde

$$
x_{i}=0,000125+(0,0001875)(i-1), i=1, \ldots, 11
$$

de modo a incluir um maior número de pontos entre a superfície, $x=0$ e a espessura da placa $x_{L}=0,002(m)$. 
Figura 2 - Concentrações estimadas por $C_{p_{5}}\left(x, t_{1}=20\right)$ e $C_{S}\left(x_{i}, t_{1}=20\right)$.

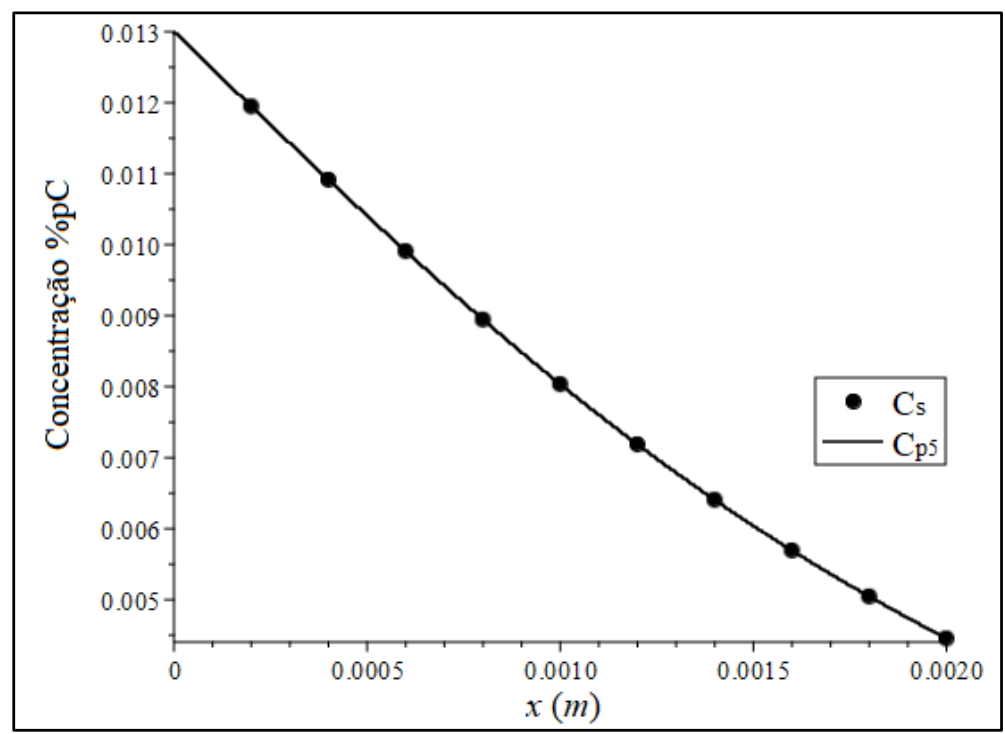

Fonte: Dados da pesquisa.

A Figura 2 mostra uma concordância entre as estimativas obtidas pelos dois métodos notando-se na vizinhança de $x_{L}=0,002(\mathrm{~m})$ uma pequena discordância devido ao limite da função de aproximação usando um polinômio de quinto grau $p_{5}(z)$. Uma vez obtido o $\operatorname{par}\left(x_{L}, t_{1}\right)$ toda a informação sobre a concentração $C\left(x, t_{1}\right)$ pode ser estimada pela função contínua $C_{p_{5}}\left(x, t_{1}\right)$ com diferenças menores que 0,004 no intervalo $0 \leq x \leq x_{L}$. Embora a função erro não possa ser resolvida analiticamente, é possível obter o fluxo de difusão através da equação (36) derivando-a em relação à variável espacial $x$ para obter

$$
\frac{d C}{d x}=\left(C_{o}-C_{1}\right) \frac{1}{\sqrt{\pi D t}} e^{-\frac{x^{2}}{4 D t}}
$$

O fluxo difusivo, $\phi(x, t)$ no ponto $x$ para um dado instante de tempo $t$ pode ser expresso segundo Churchill (1963, p. 11) como

$$
\phi(x, t)=-D \frac{d C}{d n}
$$

onde $n$, é o vetor normal unitário à superfície no ponto $x$. Das equações (45) e (46) resulta que

$$
\phi(x, t)=D\left(C_{1}-C_{0}\right) \frac{1}{\sqrt{\pi D t}} e^{-\frac{x^{2}}{4 D t}}
$$

A Figura 3 mostra o perfil do fluxo de carbono no interior da placa metálica nas posições para $x=0,001 x=0,0015$ e $x=0,0020(m)$ abaixo da superfície. O fator $C_{1}-C_{0}$ da equação (47) originalmente dado em percentual em peso de carbono é transformado em $\mathrm{Kg} / \mathrm{m}^{3}$ por meio de relações de conversão estabelecidas por Callister (2002). 
Figura 3 - Perfil do fluxo de difusão em diversas profundidades da placa em metros.

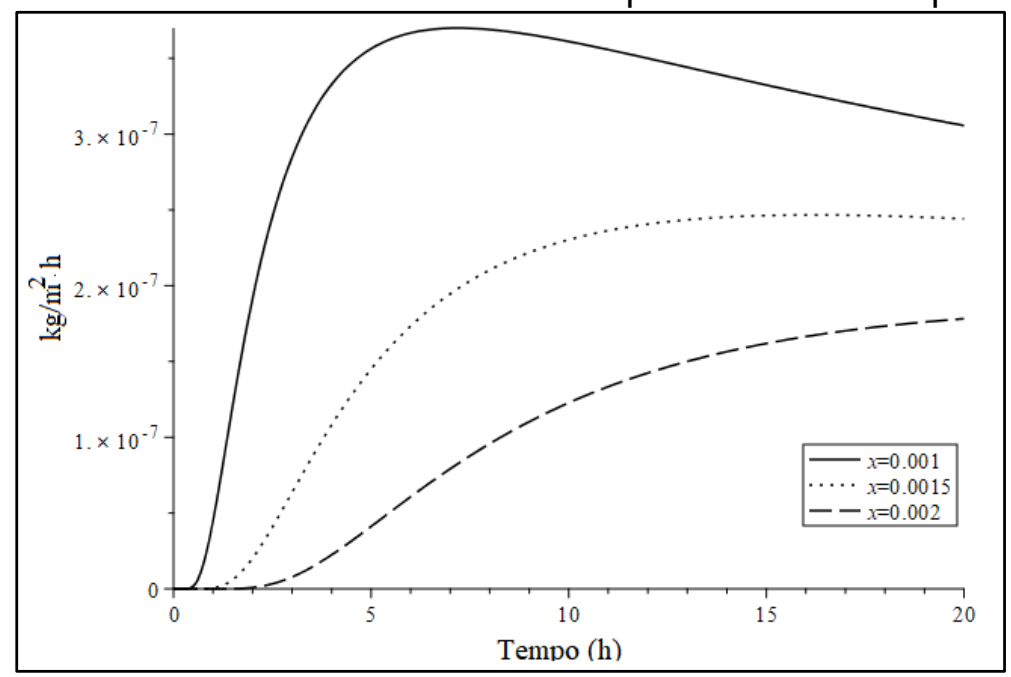

Fonte: Dados da pesquisa.

Como era de se esperar o processo de difusão é mais intenso perto da superfície, e após aproximadamente 7 horas o fluxo difusivo começa a diminuir na região situada em $x=0,001(\mathrm{~m})$ abaixo da superfície. Embora na maioria dos problemas em engenharia as soluções numéricas sejam as mais utilizadas devido à impossibilidade de soluções analíticas ou mesmo, a dificuldade em obtê-las através do formalismo matemático exigido, a solução exata deve ser preferível, mesmo que seja complicada em oposição a soluções numéricas, pois dependendo do grau de acuidade exigido pelo problema, o procedimento numérico poderá demandar um elevado esforço computacional, isso quando não apresentar problemas com a convergência. Nesse sentido, o presente estudo mostrou que a solução formal para o problema proposto, aproximada por uma função analítica de fácil tratamento matemático concordou, dentro de certos limites obtidos de forma empírica do domínio espacial, com a solução utilizando métodos numéricos para a representação de estimativas de concentração de carbono no interior da superfície da placa exposta à cementação. A metodologia exposta neste estudo também pode ser estendida a outros solutos em processo de difusão dentro das condições iniciais e de fronteira mais gerais.

\section{Conclusões}

A equação de difusão em regime transiente unidimensional utilizada neste estudo na representação de um processo de cementação em uma placa delgada homogênea de aço-carbono com as condições inicial e de fronteira dadas, pode ser resolvida detalhadamente à luz da transformada de Fourier em seno e da integração complexa. Para evitar o uso de tabelas ou métodos numéricos na resolução aproximada da função erro de Gauss, a qual aparece na solução formal da equação de difusão, um polinômio de quinto grau foi utilizado em substituição ao uso do cálculo da função erro de Gauss. Os resultados do processo na amostra analisada utilizando a solução formal da equação de difusão com a função erro de Gauss e o uso do polinômio de quinto grau ficaram em boa concordância. 
Tais resultados indicam que essa metodologia alternativa em um domínio espacial, embora restrito, pode ser usada na análise de estimativas para a concentração de outros solutos em processo de difusão por diferença do gradiente de concentração e elevada temperatura dentro de condições iniciais e de fronteira mais gerais.

\section{Referências}

ARAUJO, J.; MÁRQUEZ, R. Análise de Temperaturas em uma Barra Uniforme de Aço-Carbono com o Método Explícito. C.Q.D. Revista Eletrônica Paulista de Matemática. v. 2, n. 2, p. 58-69, 2013.

BOYCE, W. E.; DIPRIMA, R. C. Equações diferenciais elementares e problemas de valores de contorno. 6. ed. Rio de Janeiro: LTC, 1999.

BROWN, K. W.; CHURCHILL, R. V. Variáveis complexas e aplicações. 9. ed. Porto Alegre: McGraw Hill Education, 2015.

CALLISTER, W. D. Jr. Ciência e Engenharia de Materiais: Uma Introdução. 5. ed. Rio de Janeiro: LTC, 2002.

CARSLAW, H. S.; JAEGER, J. C. Conduction of Heat in Solids. 2. ed. Clarendon Press-Oxford, 2011.

CASTRO, A. S. Estados ligados em um potencial delta duplo via transformadas seno e cosseno de Fourier. Revista Brasileira de Ensino de Física, São Paulo, v. 36, n. 2, p. 1-5, 2014.

CHURCHILL, R. V. Fourier Series and Boundary Value Problems. 2. ed. New York: McGrawHill, 1963.

CRANK J. The Mathematics of Diffusion. 2. ed. Clarendon Press-Oxford, 2011.

DEBNATH, L.; BHATTA, D. Integral transforms and their applications. 3. ed. Boca Raton: A Chapman \& Hall Book, 2015.

GARZÓN C. M.; TSCHIPTSCHIN, A. P. Modelamento Termodinâmico e Cinético por meio do Método Calphad do Processamento Térmico e Termoquímico de Aços. Revista Matéria. v. 11, n. 2, p. 70-87, 2006.

IÓRIO, V. EDP: um curso de graduação. Rio de Janeiro: IMPA, 1991. (Coleção Matemática Universitária).

LIMA, E. L. Curso de Análise. v.1. 14. ed. Rio de Janeiro: IMPA, 2016.

NEGERO, N. T. Fourier transform methods for partial differential equations. International Journal of Partial Differential Equations and Applications, v. 2, n. 3, p. 44-57, 2014.

RUGGIERO, M. A. G.; LOPES, V. L. R. Cálculo Numérico: Aspectos Teóricos e Computacionais. 2. ed. Pearson Makron Books, 2006.

TIJONOV, A.; SAMARSKY, A. Ecuaciones de la Física Matemática. 2. ed. Editoral MIR, Moscu, 1980.

TRIM D. W. Applied Partial Differential Equations. Boston: PWS-KENT Publishing Company, 1990. 